\title{
ASO Author Reflections: APRI + ALBI: A Novel Tool for Estimating Chemotherapy-Associated Liver Injury in Patients with Colorectal Cancer Liver Metastasis Undergoing Liver Resection
}

\author{
D. Pereyra, and P. Starlinger, MD, PhD \\ Department of Surgery, General Hospital, Medical University of Vienna, Vienna, Austria
}

\section{PAST}

Within the past decade, the management of patients treated for colorectal cancer liver metastasis changed rapidly and evolved to be one of the most prominent fields within surgical oncology. Perioperative chemotherapy improves the outcome of both resectable and initially unresectable colorectal cancer liver metastasis. ${ }^{1}$ Despite the benefit in terms of oncologic outcome, chemotherapyassociated liver injury is frequently observed in these patients and shown to have a significant negative impact on the postoperative clinical outcome after liver resection., ${ }^{2,3}$ This is of specific relevance because postoperative morbidity significantly affects the overall oncologic outcome of colorectal cancer liver metastasis. Yet, to date, no valid tool exists to assess chemotherapy-associated liver injury. Therefore, preoperative risk stratification after neoadjuvant chemotherapy remains a difficult task for clinicians worldwide.

ASO Author Reflections offer a brief invited commentary on the article, The combination of APRI and ALBI Facilitates Preoperative Risk Stratification for Patients Undergoing Liver Surgery After Neoadjuvant Chemotherapy. Ann Surg Oncol. 2019;26:791.

\section{(c) The Author(s) 2019}

First Received: 12 April 2019;

Published Online: 29 April 2019

P. Starlinger, MD, $\mathrm{PhD}$

e-mail: patrick.starlinger@meduniwien.ac.at

\section{PRESENT}

This study aimed to define a tool to assess chemotherapy-associated liver injury after neoadjuvant chemotherapy. ${ }^{4}$ The study focused particularly on patients with colorectal cancer liver metastasis undergoing liver resection. The marker chosen was to be based on routine laboratory data and easily applicable within the routine clinical setting. Furthermore, the evaluated marker was not only to be associated with chemotherapy-associated liver damage, but also to be a sensitive predictor of deteriorated postoperative outcome. In this context, APRI + ALBI, a composite of aspartate aminotransferase, platelet count, albumin, and bilirubin, was found to be increased in patients displaying chemotherapy-associated liver injury. Moreover, an increase after completion of neoadjuvant chemotherapy was observed. Ultimately, APRI + ALBI was found to facilitate preoperative risk stratification in patients after neoadjuvant chemotherapy via identification of patients more likely to experience postoperative liver dysfunction and morbidity.

\section{FUTURE}

In summary, APRI + ALBI allows estimation of chemotherapy-associated liver damage over time. This represents a unique property because to date no noninvasive tool exists to monitor liver damage after neoadjuvant chemotherapy. Furthermore, the findings showed that APRI + ALBI dynamically monitors liver damage because it declined within the chemotherapy-free interval before the operation, presumably reflecting gradual liver function recovery. In addition, given the association with postoperative outcome, APRI + ALBI can be used to identify patients experiencing clinically relevant 
chemotherapy-associated liver injury. Ideally, this marker, which was not only established but also validated in a multicenter fashion, will be used as a tool for personalized scheduling of liver resection after neoadjuvant chemotherapy. Patients exceeding the proposed cutoff before the operation might benefit from postponement of liver resection until liver function has further improved. This might eventually lead to a reduction in postoperative complications and hence improvement of the overall outcome for this specific patient cohort.

ACKNOWLEDGMENTS Open access funding provided by Medical University of Vienna.

DISCLOSURE The authors have no conflicts of interest to disclose.

OPEN ACCESS This article is distributed under the terms of the Creative Commons Attribution 4.0 International License (http://crea tivecommons.org/licenses/by/4.0/), which permits unrestricted use, distribution, and reproduction in any medium, provided you give appropriate credit to the original author(s) and the source, provide a link to the Creative Commons license, and indicate if changes were made.

\section{REFERENCES}

1. Nordlinger B, Sorbye H, Glimelius B, et al. Perioperative chemotherapy with FOLFOX4 and surgery versus surgery alone for resectable liver metastases from colorectal cancer (EORTC Intergroup trial 40983): a randomised controlled trial. Lancet. 2008;371:1007-16.

2. Tamandl D, Klinger M, Eipeldauer S, et al. Sinusoidal obstruction syndrome impairs long-term outcome of colorectal liver metastases treated with resection after neoadjuvant chemotherapy. Ann Surg Oncol. 2011;18:421-30.

3. Zhao J, van Mierlo KMC, Gomez-Ramirez J, et al. Systematic review of the influence of chemotherapy-associated liver injury on outcome after partial hepatectomy for colorectal liver metastases. Br J Surg. 2017;104:990-1002.

4. Pereyra D, Rumpf B, Ammann M, et al. The combination of APRI and ALBI facilitates preoperative risk stratification for patients undergoing liver surgery after neoadjuvant chemotherapy. Ann Surg Oncol. 2019; 26:791.

Publisher's Note Springer Nature remains neutral with regard to jurisdictional claims in published maps and institutional affiliations. 